\title{
Visualization of Salt-Induced Stress Perturbations
}

\author{
Patricia Crossno ${ }^{\dagger}$ \\ Sandia National Laboratories \\ David H. Rogers ${ }^{\ddagger}$ \\ Sandia National Laboratories
}

\author{
Rebecca M. Brannon* \\ Sandia National Laboratories
}

\author{
David Coblentz ${ }^{\S}$ \\ Los Alamos National \\ Laboratories
}

\begin{abstract}
An important challenge encountered during post-processing of finite element analyses is the visualizing of three-dimensional fields of real-valued second-order tensors. Namely, as finite element meshes become more complex and detailed, evaluation and presentation of the principal stresses becomes correspondingly problematic.

In this paper, we describe techniques used to visualize simulations of perturbed in-situ stress fields associated with hypothetical salt bodies in the Gulf of Mexico. We present an adaptation of the Mohr diagram, a graphical paper and pencil method used by the material mechanics community for estimating coordinate transformations for stress tensors, as a new tensor glyph for dynamically exploring tensor variables within threedimensional finite element models. This interactive glyph can be used as either a probe or a filter through brushing and linking.
\end{abstract}

CR Categories: I.3.8 [Computer Graphics]: Applications.

Keywords: tensor field visualization, Mohr's circles, visual debugging, finite element codes and simulations.

\section{INTRODUCTION}

Tensors play an important role in many types of physically- based simulations, including velocity gradients in fluid flow, stress analysis in materials mechanics, and geomechanical modeling in oil and gas exploration. Consequently, we are concerned with visualizing three-dimensional fields of real-valued second-order tensors.

The motivation for our work is to present tensor information in a way that taps into our users pre-existing mental models. Since our users are predominantly from the material mechanics and geomechanics communities, they have requested that we base our visualization on the Mohr diagram, which is a paper-andpencil graphic aid that they learned as undergraduates in introductory courses. Although we have modernized the Mohr diagram by adding interactivity, and extended it to encode additional information about groups of tensors, we have retained enough of the original concept for our users to easily understand the visualization.

Our task hinges primarily on the visualization of symmetric tensors because any general tensor can be decomposed additively into symmetric and skew-symmetric parts, or alternatively, multiplicatively decomposed into the product of an orthogonal (unitary) rotation tensor and a symmetric (stretch) tensor. Both skew-symmetric and orthogonal tensors may be visualized through standard vector visualization tools (for example, the skew-symmetric part of a velocity gradient is proportional to the

\footnotetext{
†e-mail: pjcross@sandia.gov, ${ }^{\ddagger}$ e-mail: dhroger@sandia.gov

*e-mail: rmbrann@sandia.gov, §e-mail: coblentz@lanl.gov
}

IEEE Visualization 2004

October 10-15, Austin, Texas, USA

0-7803-8788-0/04/\$20.00 @2004 IEEE vorticity vector field; also, though rotation operations do not commute, any rotation tensor can be quantified by a pseudorotation vector through application of the Euler-Rodrigues theorem [8]).

The tensors examined in this paper are stress tensors, which can be decomposed into a symmetric part representing local force balance plus an anti-symmetric part balancing distributed torques (which are usually zero for the vast majority of engineering applications, making stress typically symmetric).

We define a second-order tensor $\mathbf{T}$ to be a $3 \times 3$ matrix of values relative to a given "physical” basis:

$$
\mathbf{T}=\left[\begin{array}{lll}
t_{11} & t_{12} & t_{13} \\
t_{21} & t_{22} & t_{23} \\
t_{31} & t_{32} & t_{33}
\end{array}\right]
$$

Any tensor $\mathbf{T}$ can be decomposed as $\mathbf{T}=\mathbf{S}+\mathbf{A}$, where $\mathbf{S}$ is symmetric $\left(S_{\mathrm{ij}}=S_{\mathrm{ji}}\right)$ and $\mathbf{A}$ is skew-symmetric $\left(A_{\mathrm{ij}}=-A_{\mathrm{ji}}\right)$. There always exists an alternative basis - the principal basis - in which the off-diagonals of $\mathbf{S}$ are zero and the diagonal components equal the eigenvalues.

After diagonalization of $\mathbf{S}$, the eigenvalues are $\lambda_{1}, \lambda_{2}$, and $\lambda_{3}$, ordered so that $\lambda_{1} \geq \lambda_{2} \geq \lambda_{3}$; the corresponding orthonormalized eigenvectors are $\mathbf{e}_{1}, \mathbf{e}_{2}$, and $\mathbf{e}_{3}$. The eigenvectors are the principal axes of the tensor and are respectively known as the major, medium and minor axes. Eigenvalues and eigenvectors have profoundly useful physical meanings that vary depending on the definition of the source tensor $\mathbf{S}$. If, for example, $\mathbf{S}$ represents a stress, scaling the eigenvectors by the eigenvalues provides a measure of the forces per unit area in orthonormal directions. There are no shearing stresses on the principal planes; planes of extremum shearing stress form equal angles with two principal planes. If $\mathbf{S}$ represents the stretch tensor from a multiplicative decomposition of material deformation, an eigenvalue equals the ratio of deformed length to initial length of the material fiber parallel to the eigenvector. This paper aims to visualize eigenvalues and eigenvectors in an ensemble sense. Our lexicon treats $\mathbf{S}$ as a stress tensor, but the techniques apply equally well to any other type of symmetric tensor.

We can determine the types of forces acting on a material element by examining the signs of its tensor's eigenvalues. If the eigenvalues are all positive, then the forces are tensile, meaning that the element is elongated in tension. If the eigenvalues are all negative, the element is being compressed. If the eigenvalues are of mixed sign, then the element is compressed in some directions and pulled in others. When all of the eigenvalues are equal, $\lambda_{1}=$ $\lambda_{2}=\lambda_{3}$, the forces are said to be isotropic, and (if the material is also isotropic) the element changes size without changing shape. When the eigenvalues are unequal, the forces are anisotropic.

\section{MOHR Diagrams}

Otto Mohr developed Mohr diagrams, or Mohr's circles, around 1900 as a graphical method for performing coordinate transformations for stress. Although they were developed to analyze stress, they can be used with any tensor matrix. The method applies to both symmetric and nonsymmetric tensors in 
two dimensions. However in three-dimensions, Mohr diagrams are limited to describing just symmetric tensors [2].

For three-dimensional symmetric tensors, the Mohr diagram is actually a triad of circles. However, we will refer to the entire triad as a Mohr's circle, since each triad represents a single tensor. The Mohr diagram is generated by first performing an eigenvalue decomposition of the tensor. For stress tensors, the eigenvectors are normal to planes that are subjected to only normal stress (zero shear). However, planes not aligned with the principal directions generally experience both normal and shearing stresses. Consequently, there exists a state space of shear stress, $\tau$, versus normal stress, $\sigma$, characterizing achievable solutions over the infinity of possible orientations of an arbitrary plane cutting through the element. Otto Mohr proved that the set of achievable pairs of $\tau$ and $\sigma$ would always fall within the interior of a circle with diameter equal to the difference between largest and smallest eigenvalues; furthermore, attainable stress pairs will always fall outside the circles formed by differences between the extreme and middle eigenvalues. For symmetric tensors, these circles will always fall on the normal stress axis, so constructing them is simply a matter of drawing three circles between the eigenvalues. Of course, these circles must fall on the $\sigma$ axis because principal planes suffer no shear.

The outer circle is a measure of the degree of anisotropy of the tensor; the larger the circle, the more anisotropic the tensor is (i.e., the greater the peak attainable shear stress). Isotropic tensors collapse to a point, meaning that there is no shear stress regardless of plane orientation. The position of the Mohr's circle on the $\sigma$ axis relative to the origin represents whether the tensor is in compression or tension. Circles that are entirely to the left of the origin are in compression; those to the right are in tension. Circles enclosing the origin represent tensors with a combination of both compressive and tensile forces.

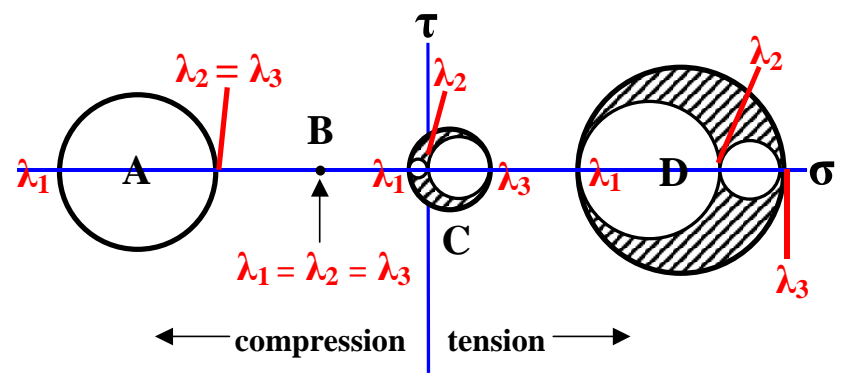

Figure 1. Mohr's circles examples.

In Figure 1, there are four Mohr's circles (each illustrating different stress tensors) lettered A, B, C, and D. Both A and B represent compression tensors. $\mathrm{D}$ is a tensile tensor. $\mathrm{C}$ has a combination of both compressive and tensile forces. All of the tensors are anisotropic, except for B, which is isotropic. The diameter of the outer circle shows the spread between the major and the minor eigenvalues, $\lambda_{1}$ and $\lambda_{3}$. The intersection of the two smaller inner circles shows the value for the medium eigenvector, $\lambda_{2}$. Note that in $A, \lambda_{2}=\lambda_{3}$, so that the larger of the two inner circles overlaps the outer circle and the smaller inner circle goes to a point. Both $\mathrm{A}$ and $\mathrm{B}$ represent degenerate cases used to identify features [17]. The shaded regions in C and D show all the achievable solutions for $\tau$ and $\sigma$ within tensors $C$ and $\mathrm{D}$.

\section{RELATED WORK}

Most previous work in tensor visualization falls into one of several categories: glyphs, feature-based, art-based, volume rendered, and deformations. Since volume rendered and deformation approaches bear little similarity to our work, they will not be described further.

A number of glyphs have been developed for viewing tensor data. The most common and intuitive glyph is the ellipsoid, which is drawn using the tensor's eigenvectors to form the principal axes and the eigenvalues to scale the ellipsoid along those axes [15] [20]. Although color-coding sub-regions of ellipsoids to show eigenvalue sign has been tried [26], ellipsoids are generally limited to symmetric, real, positive tensors where all of the eigenvalues are positive [22], so they are typically not used to visualize stress fields. They are good for visualizing diffusion and stretch tensors, though they do have some drawbacks. For data sets where the diffusion rates vary greatly, the smaller ellipsoids virtually disappear [16]. Plus, rendering ambiguities can make it difficult to distinguish between a flattened ellipsoid and a sphere when viewed face-on [25]. To overcome this problem, Westin, et al. [25] proposed a glyph that is the union of a sphere, a disk and a rod, the relative size of each encoding a different eigenvalue.

The Haber glyph [9] is composed of a bar drawn along the principal eigenvector impaling an elliptical disk representing the other eigenvector directions, scaled by the respective eigenvalues. The principal stress hedgehog [13] simply draws the eigenvectors as orthonormal axes scaled by their eigenvalues and colored by eigenvalue sign (red in tension, green in compression). Livingston [18] uses two glyph types, cylindrical tufts and axis tripods, combined with animation and/or depth cueing to visualize rotation fields. The Reynolds glyph is peanut-shaped and because the distance from the origin of the glyph surface is determined by the magnitude of the normal stress in that direction, the glyph emphasizes the anisotropy of the tensor [15]. VisCoRe [10] is a framework for visualizing material constitutive relations in geotechnical engineering that uses animated three-dimensional Reynolds glyphs. However, all of these glyphs quickly occlude one another in three-dimensional fields for models of any size or complexity, so they are best when used only on two-dimensional data sets.

Probes overcome the clutter problem by interactively moving the glyph through the model and changing it to reflect the local tensor characteristics. De Leeuw and van Wijk's probe [5] [20] for viewing the velocity gradient tensor in flow fields is one wellknown example. It uses a complex icon consisting of an arrow and multiple flexible disks to represent local quantities such as velocity, curvature and shear. Sigfridsson et al.'s [22] hybrid technique combines texture-based volume rendering with a single ellipsoid glyph to provide continuous context with detailed tensor information in a region of interest.

Delmarcelle and Hesselink’s hyperstreamline glyphs [6] [15] are a type of regional probe. A path is advected through the tensor field along one of the eigenvectors, while at each step a tubular surface based on the magnitude and orientation of the other two eigenvectors is drawn. Rather than representing the value of a single tensor, the trumpet-shaped glyph represents a continuum of stresses along the trajectory. Hesselink et al. [12] [17] generated topological skeletons within tensor fields by locating singularities and connecting them with hyperstreamlines integrated along the separatrices. However, even this approach can become cluttered if there are too many hyperstreamlines in the same image. Weinstein et al. [24] developed tensorlines as an alternative feature-following method that better handles propagation through isotropic regions by including nearby orientation information in the path calculation.

Kirby et al. [14] present an artistically-based approach that encodes seven attributes of diffusion tensors as different colored layers and types of simulated brush strokes to visualize a twodimensional slice of a mouse spinal cord. Laidlaw et al. [16] 
apply this technique using layered arrows, ellipses and color to simultaneously visualize multiple attributes in a two-dimensional simulation of flow around a post. In both of these papers, the various brush strokes, or the arrows and ellipses, can be thought of as complex, layered glyphs that provide different information when viewed at different distances.

Although Mohr diagrams are well known within the material mechanics and geomechanics communities, they are virtually unknown within the visualization community. The only mention of Mohr's diagrams that we have found in the visualization literature was in VizCoRe [10], where Mohr's circles are used to represent stress state while doing stress element analysis. However, it appears that their implementation of Mohr's circles is static and does not include interactive features, such as brushing and linking. Some general advantages to using Mohr diagrams are that they can be applied to tensors with negative eigenvalues [2], and they provide insight into the local tensor behavior by showing the shear (anisotropy), compression, and tensile forces.

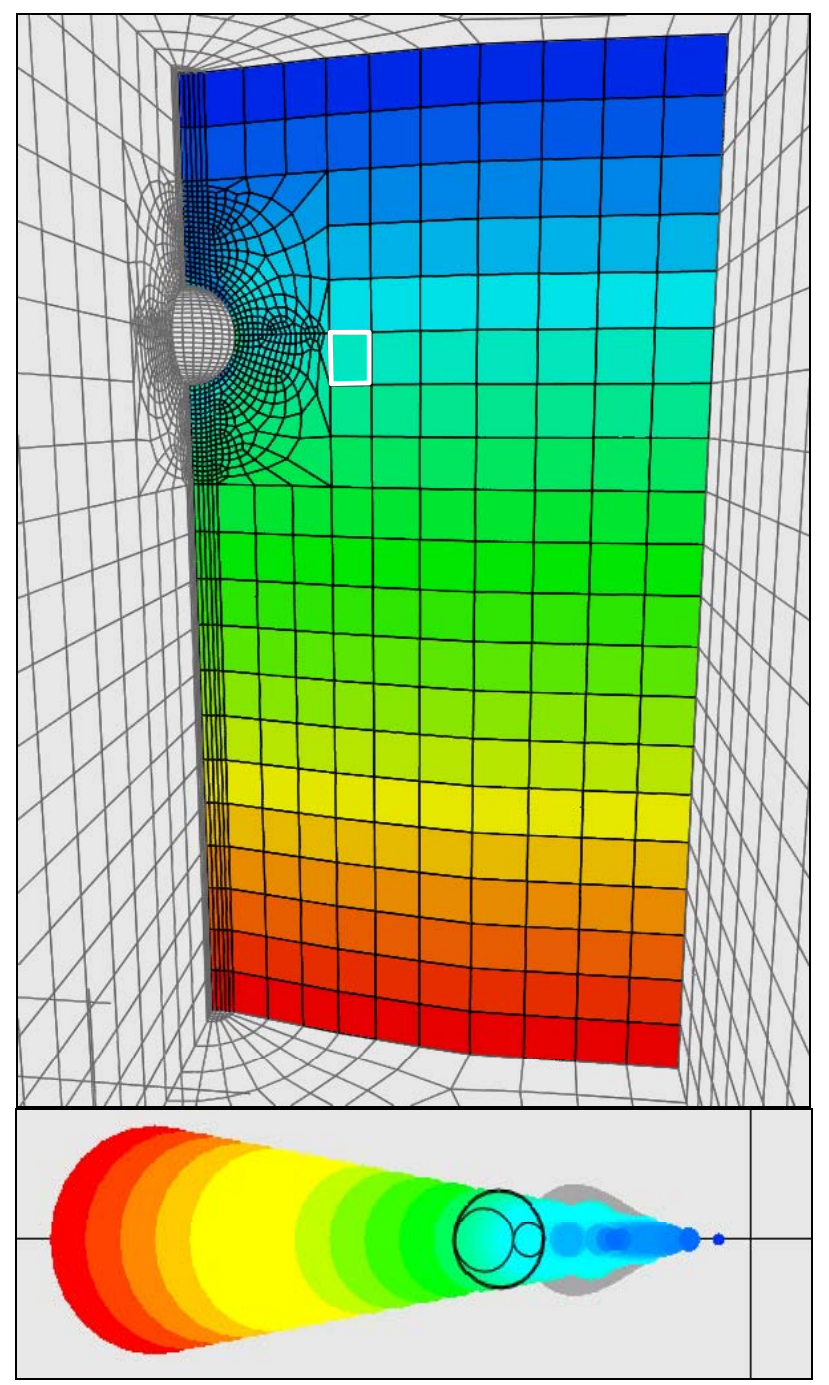

Figure 2. The upper image shows a planar subset of finite elements combined with the external faces of the model's material boundaries for context. The elements are color-coded according to the values of the displayed variable. The lower image shows the Mohr's circles for the entire subset, color-coded to correspond to the model view. The black Mohr's circle overlaying the colored circles is an interactive probe that updates as the mouse moves over the elements in the upper window.

\section{IMPLEMENTATION OVERVIEW}

This work adds tensor variables to the suite of tools we previously developed for visual debugging of finite element codes [4] [3], which had been limited to scalar and vector data types. In contrast to the previous implementation [4], all of the current functionality resides in the viewer portion of the system. So far, the geologic simulations we have been working with are small enough to be viewed in their entirety, so mesh subsets and the parallel implementation of the mesh extraction code have not been needed. The viewer is implemented in $\mathrm{C}++$ using OpenGL as the graphics API. The user interface is written in Tcl/Tk.

The viewer displays the finite element model in one window and the Mohr diagram in another linked window as shown in Figure 2. We use brushing and linking as the interaction mechanism connecting the two windows.

In the model window, context is provided by wire frame renderings of the external faces of element groups that have significance within the model. Elements are color-coded based on the values of a user-selected element variable. To provide context in the Mohr diagram window, the Mohr's circle for a particular element can be superimposed over color-coded layers providing both global and subset information, as shown in Figure 2 and the close up in Figure 3. This assists the user in developing a global understanding of the data, as well as locating those elements with the largest degree of anisotropy or isotropy, or the greatest compressive or tensile forces.

For nonsymmetric tensors, we visualize the symmetric part using Mohr's circles and the non-symmetric part (from either an additive or multiplicative decomposition) using a separate hedgehog field, rendered in the model window, showing the direction of the principle axis of the rotational vector associated with the non-symmetric part, colored by its magnitude.

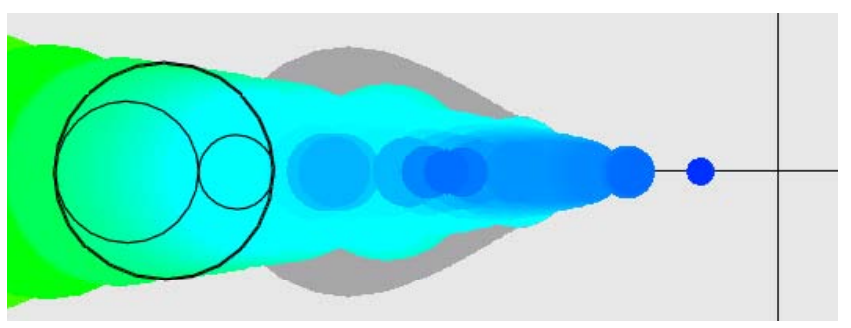

Figure 3. Zoomed-in image of Mohr's circle detail in Figure 2. The interactive Mohr's circle glyph is superimposed on both the colored subset envelope and the gray envelope showing the anisotropy for the entire model. All of the Mohr's circles are to the left of the origin, so they are all in compression.

\subsection{Contextual Information and Probing}

We expand upon the traditional Mohr diagram by including global and subset information for model elements through a series of layers that can be drawn in the Mohr diagram window. These can be viewed separately, or in combination.

First, the user can view global information drawn from the entire model. This view consists of the combined footprints from the Mohr's circles of every element in the model. This provides a filled envelope that shows the global degree of anisotropy at each position along the horizontal axis. This global envelope is drawn in gray, as a background image in the Mohr diagram window as shown in Figure 2 and Figure 3.

Additionally, the user can draw color-coded Mohr's circles for a subset of elements over the global envelope using the same color-coding as was used to render the subset of elements in the model window. An example of this overlay is shown in Figure 2, 
where the colored Mohr's circles of the elements in the planar subset are drawn over the gray envelope for the entire model. Part of the underlying global envelope is still visible behind the light blue section, indicating that there are elements with greater anisotropy for that degree of compression elsewhere in the model. The circles are drawn in order of size, from large to small, so that the smaller, darker blue circles corresponding to the upper rows of the subset plane remain visible. Figure 3 shows a zoomed-in view of this detail.

A third overlay is generated when we probe elements in the model window for stress information by using the Mohr's circle as a dynamic glyph that interactively changes as the mouse brushes over different elements in the model window. The glyph consists of a triad of black circles drawn over the color-coded subset envelope, as shown in the lower image in Figure 2 and the zoomed in version of the same image in Figure 3. The glyph can move along the horizontal axis, changing both its overall size (anisotropy) and the relative proportions of the three circles as the eigenvalues shift relative to each other.

Upon reading in a new data set, the viewer calculates the eigenvalues and eigenvectors for each tensor. The center point for each Mohr's circle is calculated as needed and the circles are sorted, first according to their size, then according to their position along the compression/tension (horizontal) axis. The circle extrema, circles with the largest and smallest sizes and the leftmost and rightmost coordinates along the horizontal axis, are found. These circles are the elements whose tensors represent the extremes in the anisotropic and/or isotropic distribution of forces, and the extremes in compressive and/or tensile forces, respectively. An example of the four Mohr's circle extrema and their associated elements is given in Figure 4. The Mohr's circles for the two red elements are so close to each other that they overlap. They can be seen as the largest, leftmost circle at the bottom of Figure 4.

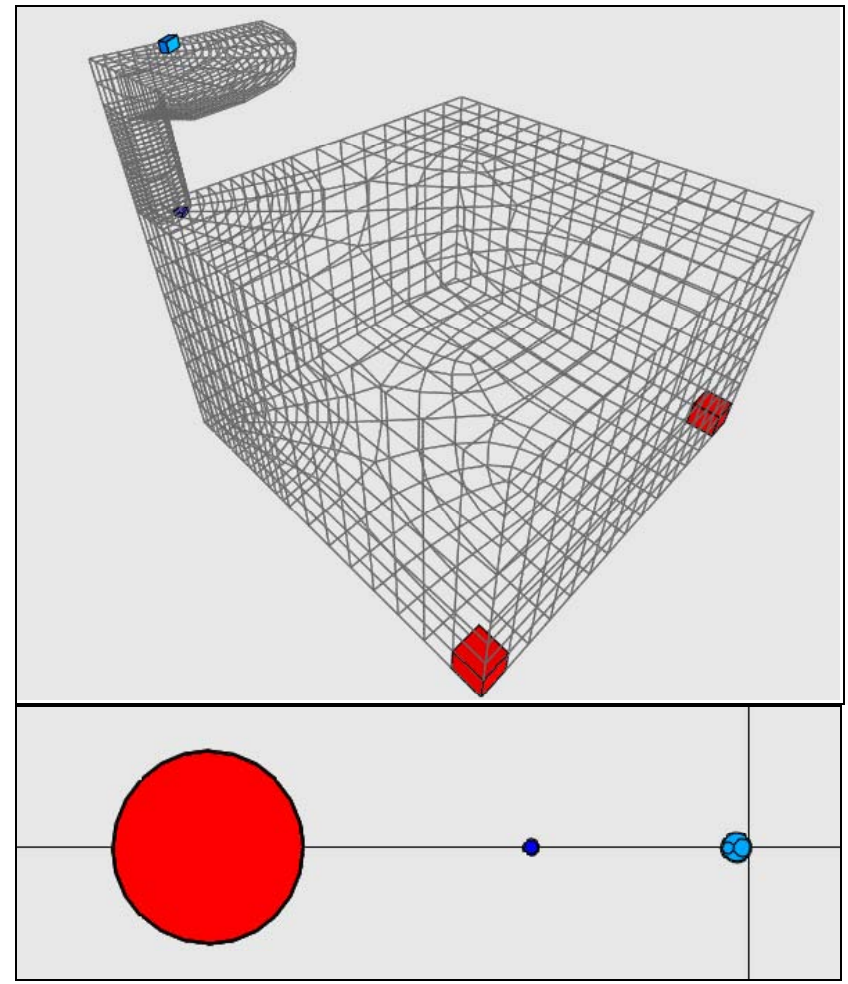

Figure 4. The four Mohr's circle extrema and their associated elements within the model window are shown. Note that the red elements produce overlapping circles in the Mohr diagram window.

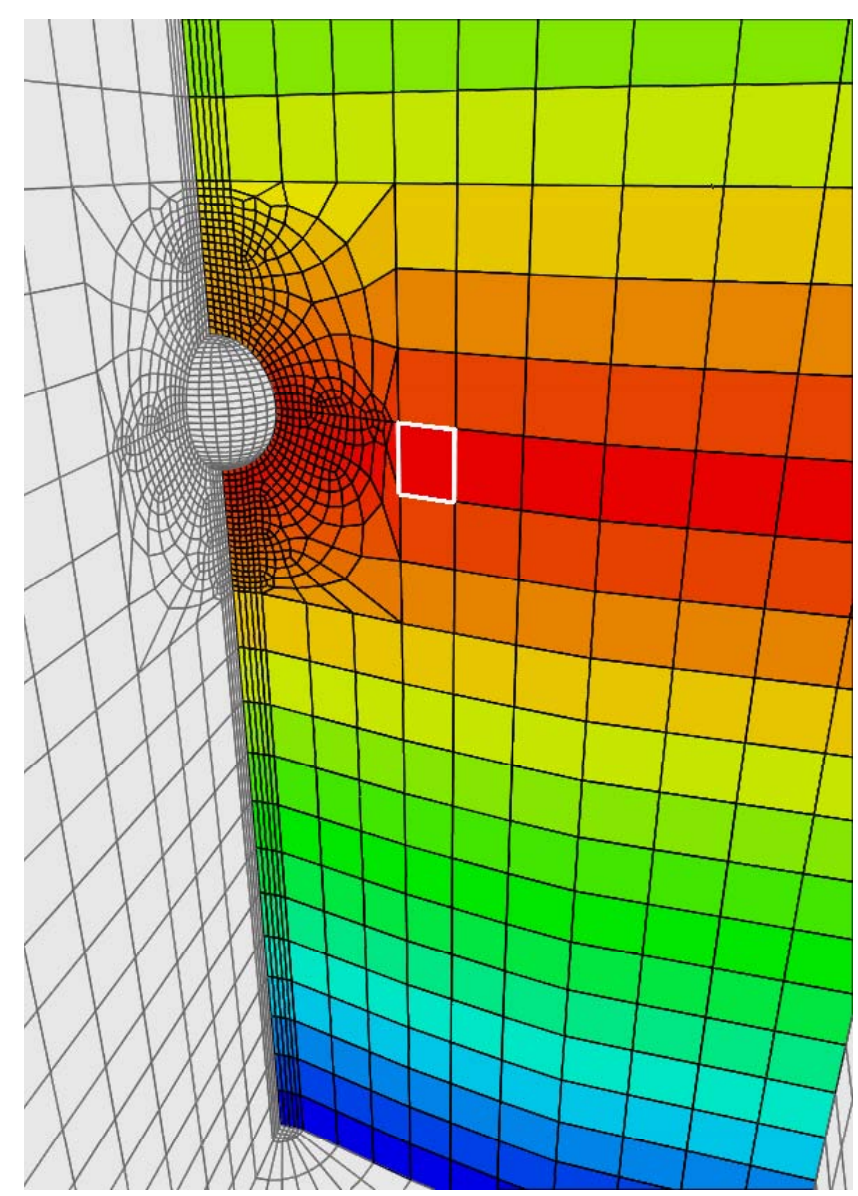

Figure 5. Elements are color-coded by similarity to the selected element, which is highlighted in white. Red elements are most similar, while blue elements are least similar.

Clicking on an element within the model window selects it. A new variable can then be created to represent the distance between the eigenvalues of the selected element and the eigenvalues of the other elements in the model. We compute the distances by treating each element's eigenvalue triplet as a point in threespace, then calculating the Euclidean distance from each point to the selected cell's point. Re-coloring the elements by this derived variable, as in Figure 5, shows at a glance those elements that are most similar to the selected element (in red) and those that are most different (in blue).

\subsection{Filtering}

Selecting circles or specifying Mohr's circle parameters within the Mohr diagram window provides a filtering mechanism that links back to the model display window. For instance, all of the elements whose degree of compression is within some tolerance of a selected circle can be chosen, or all the elements whose outer Mohr's circle radius (and degree of anisotropy) is equal to some value can be displayed.

Alternatively, an element can be selected from within the model window and all elements whose Mohr's circle parameters are within some percentage of its parameters can be selected, as is shown in Figure 6. Filtering operations can be used to create new sets and to describe new element groups. These sets can be turned on and off interactively, or combined with built-in sets from the simulation (things like elements grouped by material type or processor identifier). These new filtering operations are performed in combination with the filtering functions we 
developed previously for scalar and vector variables. This provides a rich mechanism for querying the data set for elements that meet various criteria.

This filtering capability can be used, for example, to show only those elements in a calculation that are deforming elastically. Conversely, filtering can be used to display only elements that have yielded plastically, or even to locate inadmissibly behaving elements such as finite elements on the verge of inverting.

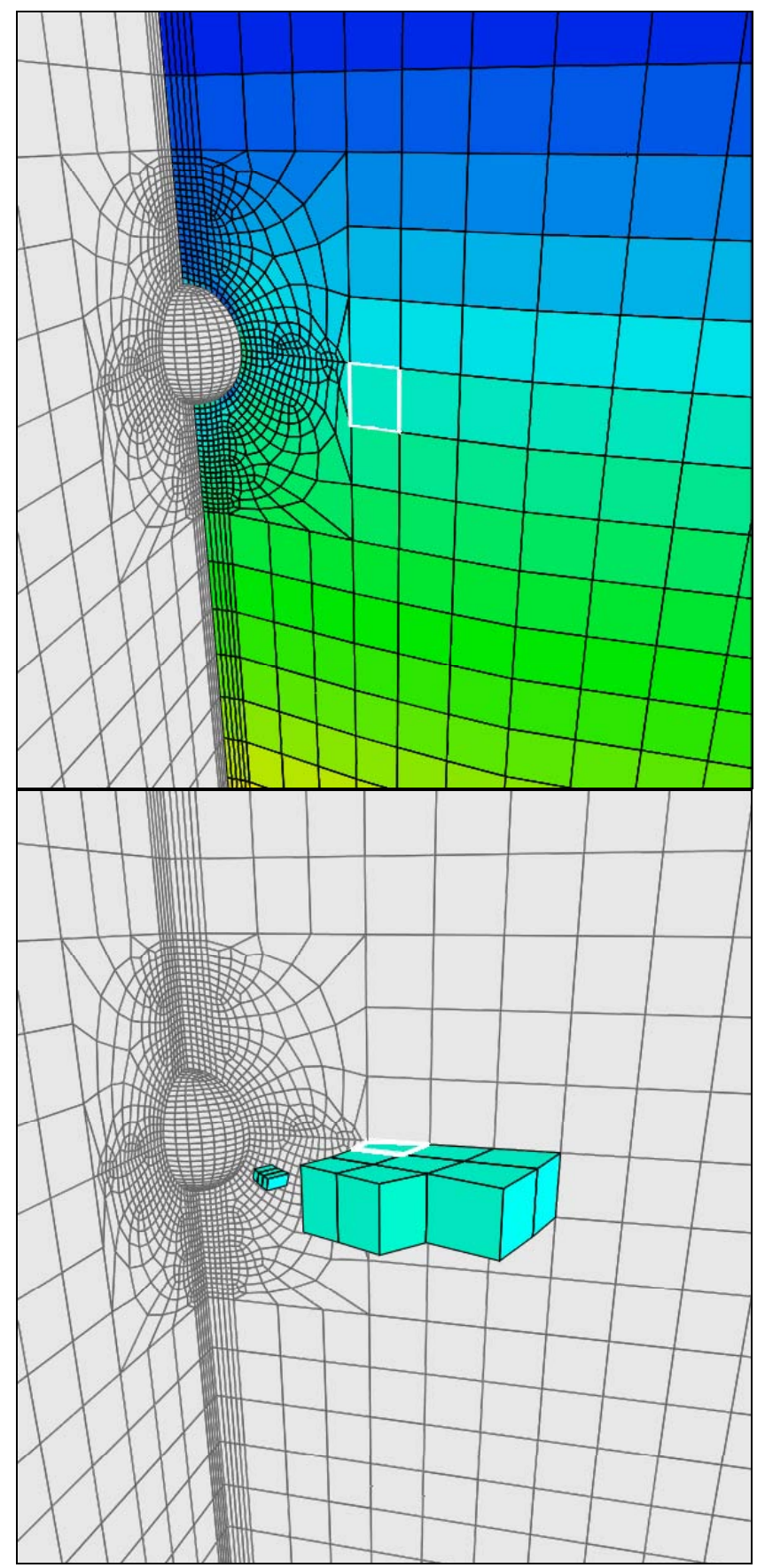

Figure 6. The selected element is outlined in white in the top image of the model window. In the lower image, the model window has been updated to display the same element along with all of the elements whose Mohr's circles fall within .2\% of its Mohr's circle parameters.

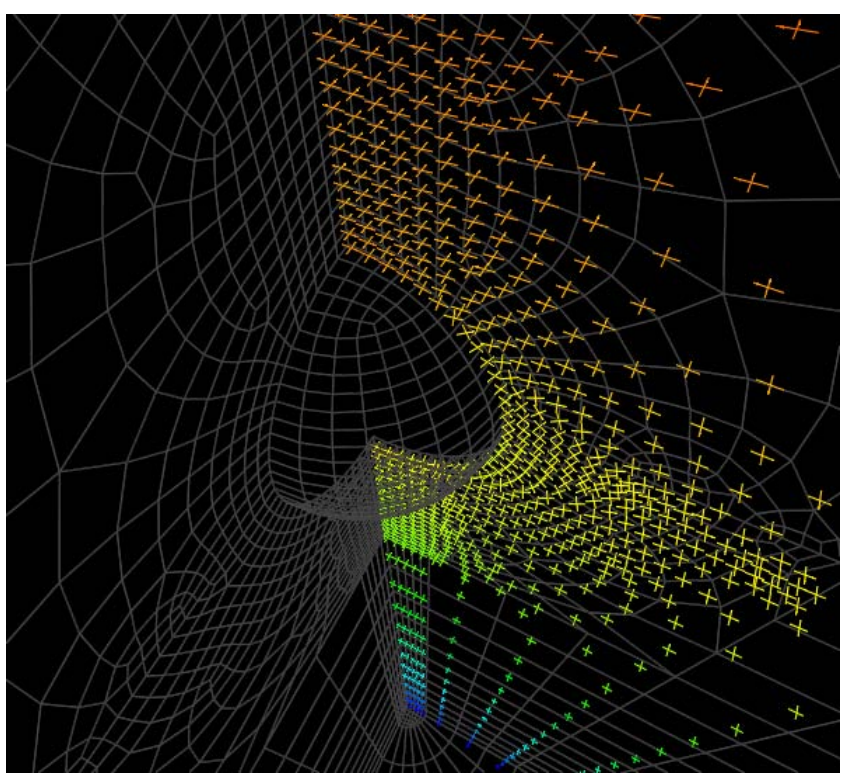

Figure 7. The principal direction vector pairs (eigenvectors) for the plane of elements shown in the upper image of Figure 6, are shown color-coded by the eigenvalues.

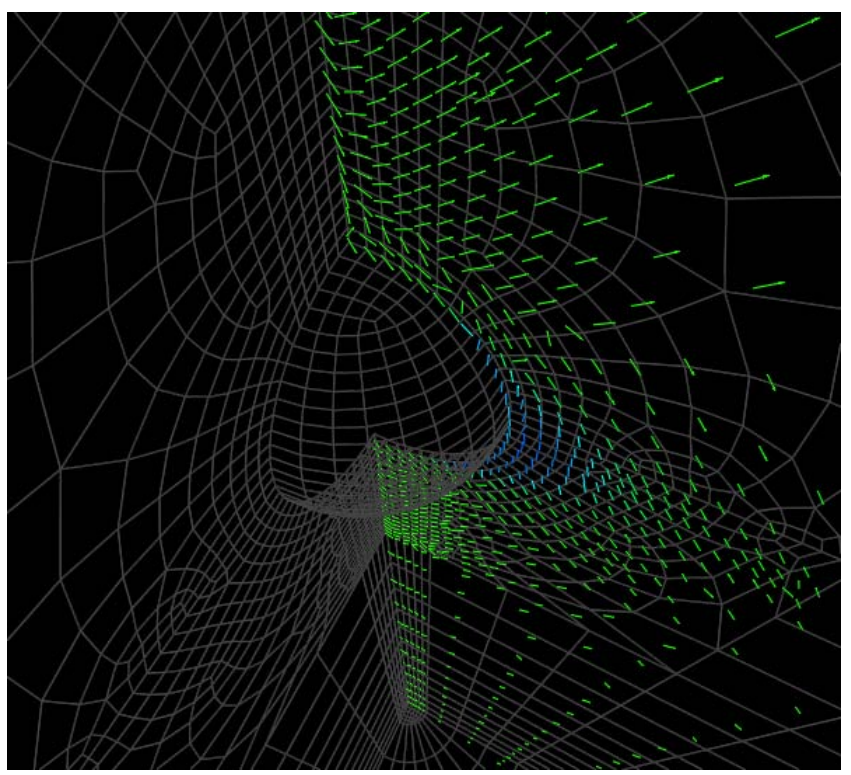

Figure 8. Axis of rotation colored by the angle of rotation. This is the alternative representation of the same information as shown in Figure 7.

The filtering of the elements in the model based on Mohr's circle parameters helps reduce clutter in a three-dimensional environment. Feature detection can be performed by filtering on either the isotropic extreme given by the Mohr's circle with the smallest radius, or on circles that have two of the three eigenvalues equal to one another [17]. This will locate the elements in the model containing degenerate points.

\subsection{Rotational Information}

For three-dimensional tensor values, principal orientations cannot be readily encoded into Mohr diagrams. Instead, we present this information in the model window. To visualize principal orientations in the viewer, the user can select from the two options 
shown in Figure 7 and Figure 8. Each of the two options was developed to support a different user community.

The option most readily understood within the mechanics community is a simple plot of the field of principal stress directions. Even though such a plot shows only two principal directions at a time, the orientation of the third eigenvector is implied by the relative orientations of the two directions that are seen. We draw the eigenvectors as a cross to reduce the visual clutter and make the image more intelligible. The user can easily understand the orientation by interactively changing the view. This option is shown in Figure 7.

The second option for visualizing principal directions in three dimensions considers the matrix of direction cosines to be a rotation matrix and applies the Euler-Rodrigues theorem to plot the axis of rotation colored by the angle of rotation. This option has considerable appeal to the geomechanics community because the direction cosine matrix can be made unique by using eigenvectors that are orthonormalized projections of the laboratory base vectors onto the eigenspaces (allowing visualization of the unique smallest rotation angle needed to transform the laboratory basis into the principal basis). Applying this to the same data as Figure 7 is demonstrated in Figure 8.

\section{Application}

The deepwater Gulf of Mexico is the most active deepwater region in the world, currently providing some of the greatest challenges in scope and opportunity for the petroleum industry. The region is estimated to contain undiscovered recoverable resources of at least 13 billion barrels of oil. However, the complexity of salt tectonics exacerbated by the extreme depths, results in high development costs and the necessity for innovative technology to successful exploit these resources.

In addition to being central to the geologic evolution of important oil and gas provinces such as the Gulf of Mexico, salt bodies affect the present-day geomechanical environment by altering the local state of stress [7]. This is fundamentally due to the fact that salt cannot sustain deviatoric stress; rather, at mean stresses above about $5 \mathrm{MPa}$, it deforms via plastic (isovolumetric) creep in response to any imposed deviatoric stress.

In passive sedimentary basins, gravitational loading drives the state of stress such that the vertical stress $S_{V}$ is due to the weight of the overburden and the horizontal stress $S_{H}$ is equal to some fraction of the vertical stress $S_{V}$ [19]. However, this state of stress cannot be sustained within salt bodies, where the stresses relax so as to reach an isotropic state of stress with $S_{H}=S_{V}$. The isotropic state of stress that exists within the salt body is at odds with the stress state in the surrounding materials that can support a deviatoric state of stress with $S_{H} \neq S_{V}$. The requirement for the salt body to be in equilibrium and to maintain continuity with the surrounding formations therefore causes the stress state near the interface to be highly complex and perturbed from the far field stress state. The only way to determine the local stress state is to solve the complete set of equilibrium, compatibility, and constitutive equations with the appropriate initial and boundary conditions.

Lack of consideration of the geomechanical interaction between salt bodies and surrounding formations has led to documented drilling failures adjacent to salt diapers [1] [21] [23], some cases resulting in individual well abandonment costs of tens of millions of dollars. To address this critical knowledge gap, a three-dimensional non-linear finite element geomechanical simulation effort was initiated to analyze the in situ stress state existing in, and adjacent to, salt bodies both before drilling, as well as under producing conditions. This work leverages unique expertise in salt mechanics and computational geomechanical modeling acquired through Sandia National Laboratories’ mission work for the U.S. Department of Energy.

The goal is to find a drilling path to the oil or gas that would reduce the shear on the well bore that often results in response to drawing down the reservoir during production. Thus far, we are only examining static configurations. We have examined two small test problems showing idealized salt structures. One data set models the salt as a sphere (Figure 2), and the other data set models the salt as a pillar (Figure 4). The salt sphere data set consists of 8,136 elements, while the salt pillar model is larger at 10,128 elements.

With each data set, we are interested in examining the tensor information for the elements near the salt formation. For the salt sphere, we have located a group of elements, shown in Figure 9, whose degree of rotation is small. Filtering the model window to display just these elements, we superimpose their Mohr's circles on the envelope (shown in gray) for the entire model. As we interactively probe the cells, the black Mohr's circle overlays the colored circles for the subset. The current element is drawn in white in the model view. Similarly, in Figure 10, we have further reduced the subset to just a plane of values through the middle of the clump shown in Figure 9. The Mohr diagram window updates to display just the color-coded circles from that plane. We continue to interactively explore plane elements. The new current element is drawn in white and its circle is overlaid in black.

In the salt pillar data set, we have filtered on those elements that display a high degree of rotation. Then we have colored coded the subset elements based on the degree of rotation and scaled by their magnitude within the selected range. This is shown in Figure 11.

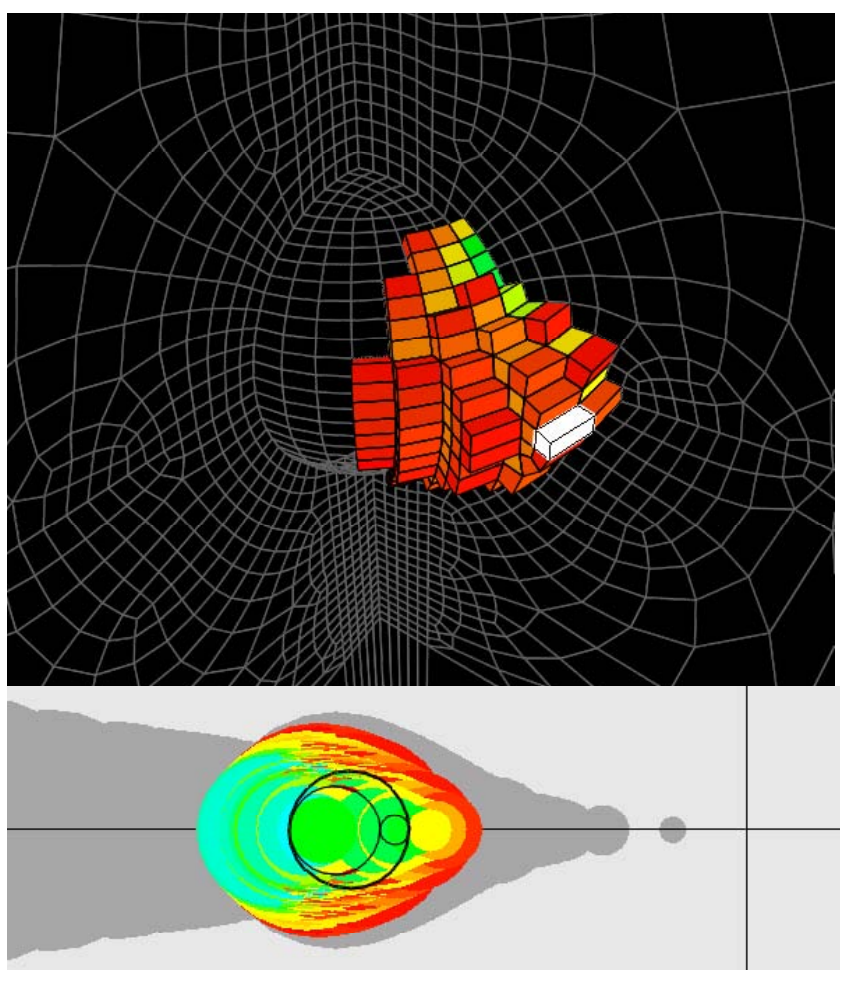

Figure 9. Filtering on the amount of rotation, we have isolated this clump of elements in the vicinity of the salt sphere. The Mohr's circles for the subset were then overlaid on the envelope from the entire model. The Mohr's circle for the element shown in white is displayed in black. 

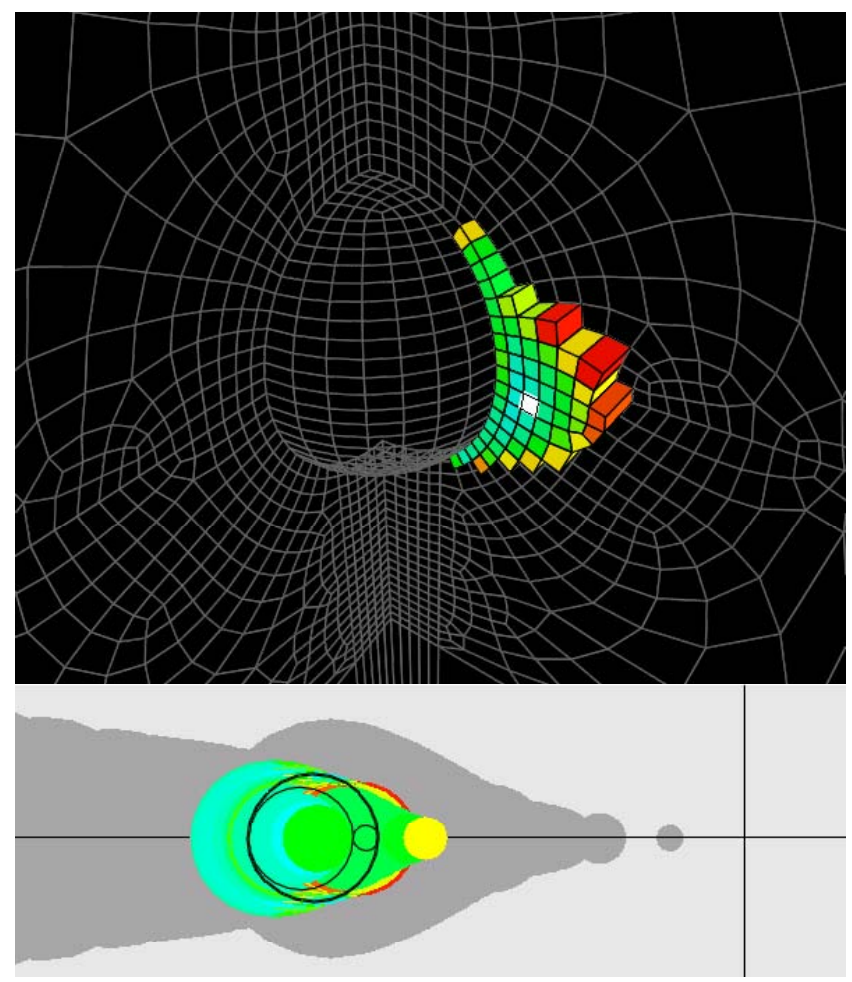

Figure 10. Planar subset of Figure 9, with Mohr's circles reflecting the current subset and selected element (in white).

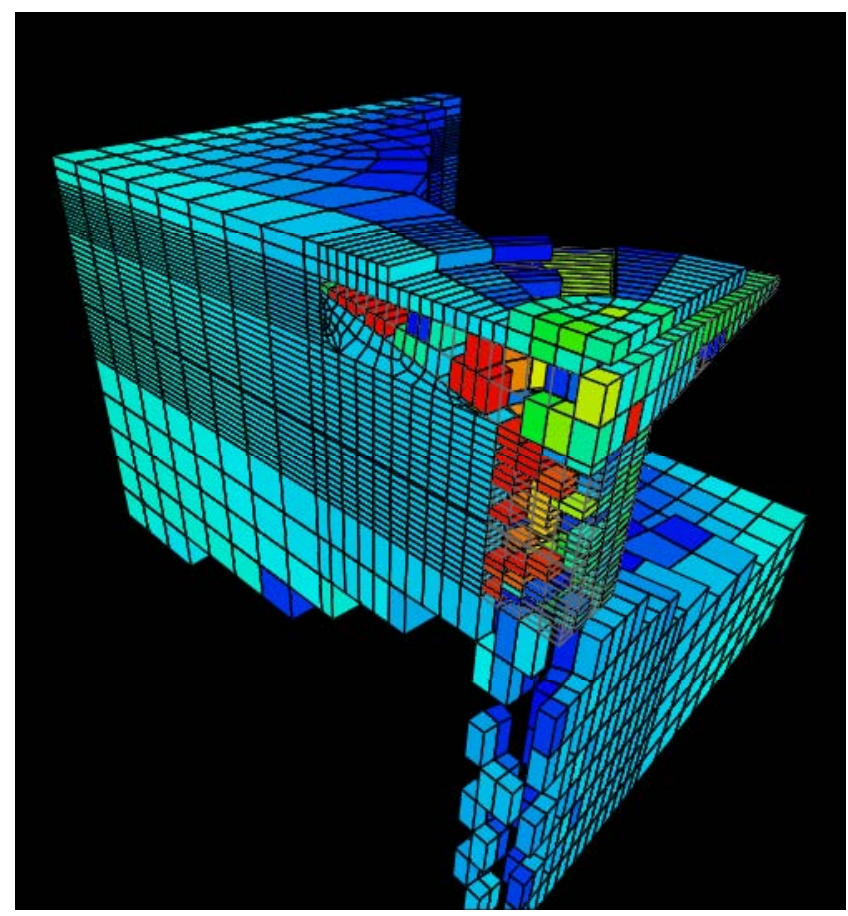

Figure 11. Salt pillar elements of highest rotation.

\section{Results}

The tensor visualization algorithm presented in this paper has helped us draw the following conclusions about the in-situ stress field perturbations near salt bodies in the Gulf of Mexico.
The isotropic state of stress that exists within salt bodies in the subsurface is at odds with the stress state in the surrounding materials that can support a deviatoric stress state with $S_{H} \neq S_{V}$. The requirement for the salt body to be in equilibrium and for it to maintain continuity with the surrounding formations causes the stress state near the salt interface to be spatially variable and perturbed from the far field stress state, including the amplification of shear stresses adjacent to salt bodies.

Three-dimensional nonlinear finite element geomechanical codes that implement sophisticated constitutive models for salt creep in conjunction with tensor visualization software can successfully quantify the character of stress perturbations within and surrounding the salt bodies.

Vertical and horizontal stresses can be significantly perturbed around (and within) salt bodies so that the vertical stress is not equal to the value that would be calculated by integration of a density log. A stress arching phenomena can naturally exist prior to drilling or production that is similar in character to that which develops in the overburden during depletion of compactable reservoirs. The common assumption that the horizontal stress within a salt body is equal to the lithostatic stress is shown for some cases to be incorrect. For some geometries, anisotropy in the horizontal stresses may be induced, which can be as high as $35 \%$ of the far field horizontal stress.

Principal stresses may rotate away from the vertical and horizontal planes close to the interface with a salt body, i.e., the vertical stress may not necessarily be the maximum principal stress. Rotations up to $20^{\circ}$ can occur in some locations.

Geomechanical modeling of the type employed here can enable more rigorous planning of well paths by providing accurate estimates of vertical and horizontal stresses around and within salt bodies for well bore stability analyses so as to avoid areas of potential geomechanical instability, and to enable accurate fracture gradient prediction while entering, drilling through, and exiting salt bodies.

\section{CONCLUSIONS AND FUTURE WORK}

The tensor visualization methods developed in this work were motivated by interviewing practicing scientists to determine what sorts of visualization tools they would like to have available. Physicists and engineers have been using Mohr's circles for the last 100 years, so an interactive visualization tool based on Mohr diagrams was immediately useful with virtually no learning curve for our material mechanics and geomechanics users. Now, this paper seeks to demonstrate the usefulness of Mohr diagrams to the visualization community by illustrating their use in a geomechanics application.

Further development of Mohr diagram visualization is already underway for advanced applications aimed at debugging finite element codes (by locating nearly inverted elements) and visualizing differences between solution methods in these codes. We are currently working on comparing solutions to the same problem generated by both Eulerian and Lagrangian implementations in order to quantify the errors introduced by each of these approaches.

\section{ACKNOWLEDGMENTS}

The Department of Energy (DOE) Mathematics, Information, and Computer Science Office funded the visualization portion of this work. An ongoing Joint Industry Partnership between the U.S. DOE and a consortium of nine oil companies funded the geomechanical section of the research. The work was performed at Sandia National Laboratories. Sandia is a multi-program laboratory operated by Sandia Corporation, a Lockheed Martin 
Company, for the United States Department of Energy's National Nuclear Security Administration under Contract DE-AC0494AL85000.

\section{REFERENCES}

[1] W. B. Bradley. Borehole Failures Near Salt Domes, paper SPE 7503 presented at 1978 Annual Fall Technical Conference and Exhibition, Houston, Oct. 1-3, 1978.

[2] Rebecca Brannon. Mohr's Circle And More Circles. http://www.me.unm.edu/ rmbrann/Mohrs_Circle.pdf, 2003.

[3] Patricia Crossno and David H. Rogers. Visual Debugging, IEEE Computer Graphics and Applications, 22(6): 6-10, 2002.

[4] Patricia Crossno, David H. Rogers, and Christopher J. Garasi. Case Study: Visual Debugging of Finite Element Codes. In Proceedings of IEEE Visualization 2002, pp. 517-520, 2002.

[5] Willem C. de Leeuw and Jarke J. van Wijk. A Probe for Local Flow Field Visualization. In Proceedings of IEEE Visualization '93, pp. 39-45, 1993

[6] Thierry Delmarcelle and Lambertus Hesselink. Visualization of Second Order Tensor Fields and Matrix Data. In Proceedings of IEEE Visualization '92, pp. 316-323, 1992

[7] Fossum, A. F. and Fredrich, J. T. Salt Mechanics Primer for NearSalt and Sub-Salt Deepwater Gulf of Mexico Field Developments. Technical Report SAND2002-2063, Sandia National Laboratories, Albuquerque, New Mexico, 2002.

[8] Herbert Goldstein, Charles P. Poole, Jr., and John L. Safko. Classical Mechanics, Columbia Univ., Addison-Wesley, 2002.

[9] R. B. Haber. Visualization Techniques for Engineering Mechanics. Computing Systems in Engineering, 1(1):37-50, 1990.

[10] Youssef M. A. Hashash, Donald C. Wotring, John I-Chiang Yao, Jung-Suk Lee and Qingwei Fu. Visual Framework for Development and Use of Constitutive Models. International Journal for Numerical and Analytical Methods in Geomechanics, Vol. 26, pp. 1493-1513, 2002.

[11] Lambertus Hesselink and Jarke J. van Wijk. Research Issues in Vector and Tensor Field Visualization. IEEE Computer Graphics and Applications, 14 (2):76-79, March 1994.

[12] Lambertus Hesselink and Jarke J. van Wijk. The Topology of Symmetric, Second-Order 3-D Tensor Fields. IEEE Transactions on Visualization and Computer Graphics, 3(1):1-11, 1997.

[13] Boris Jeremić, Gerik Scheuermann, Jan Frey, Zhaohui Yang, Bernd Hamann, Kenneth I. Joy and Hans Hagen. Tensor Visualizations in Computational Geomechanics. International Journal for Numerical and Analytical Methods in Geomechanics, Vol. 26, pp. 925-944, 2002.

[14] R. M. Kirby, H. Marmanis and David H. Laidlaw. Visualizing Multivalued Data from 2D Incompressible Flows Using Concepts from Painting. In Proceedings of IEEE Visualization '99, pp. 333340, 1999.

[15] Kriz, R. D., Glaessgen, E. H., and MacRae, J. D. EigenvalueEigenvector Glyphs: Visualizing Zeroth, Second, Fourth and Higher Order Tensors in a Continuum. Paper presented at NCSA Workshop on Modeling the Development of Residual Stresses During Thermoset Composite Curing, University of Illinois, UrbanaChampaign, September 15-16, 1995.

[16] David H. Laidlaw, Eric T. Ahrens, David Kremers, Matthew J. Avalos, Russel E. Jacobs and Carol Readhead. Visualizing Diffusion Tensor Images of the Mouse Spinal Cord. In Proceedings of IEEE Visualization '98, pp. 127-134, 1998.

[17] Yingmei Lavin, Yuval Levy and Lambertus Hesselink. Singularities in Nonuniform Tensor Fields. In Proceedings of IEEE Visualization '97, pp. 59-66, 1997.

[18] Mark A. Livingston. Visualization of Rotation Fields. In Proceedings of IEEE Visualization '97, pp. 491-494, 1997.
[19] A. McGarr and N. C. Gay. State of Stress in the Earth's Crust, Ann Rev. Earth Planet. Sci., Vol. 6, pp. 405-436, 1978.

[20] Frank J. Post, Theo van Walsum, Frits H. Post and Deborah Silver. Iconic Techniques for Feature Visualization. In Proceedings of IEEE Visualization '95, 288-295, 1995.

[21] K.P. Seymour, G. Rae, J. M. Peden, and K. Ormston. Drilling Close to Salt Diapirs in the North Sea, paper SPE 26693 presented at the Offshore European Conference, Aberdeen, September 7-10, 1993.

[22] Andreas Sigfridsson, Tino Ebbers, Einar Heiberg and Lars Wigström. Tensor Field Visualisation Using Adaptive Filtering of Noise Fields Combined with Glyph Rendering. In Proceedings of IEEE Visualization 2002, pp. 371-378, 2002.

[23] R. Sweatman, R. Faul, and C. Ballew. New Solutions for SubsaltWell Lost Circulation and Optimized Primary Cementing, paper SPE 56499 presented at the 1999 SPE Annual Technical Conference and Exhibition, Houston, October 3-6, 1999.

[24] David Weinstein, Gordon Kindlmann and Eric Lundberg. Tensorlines: Advection-Diffusion Based Propagation Through Diffusion Tensor Fields. In Proceedings of IEEE Visualization '99, pp. 249-253, 1999.

[25] C. -F. Westin, S. E. Maier, H. Mamata, A. Nabavi, F. A. Jolesz and R. Kikinis. Processing and Visualization for Diffusion Tensor MRI, Medical Image Analysis, Vol. 6, pp. 93-108, 2002.

[26] Burkhard Wünsche and Alistair A. Young. The Visualization and Measurement of Left Ventricular Deformation Using Finite Element Models, Journal of Visual Languages \& Computing, 14(4):299-326, 2003. 